\title{
12. Zusammenfassende Überlegungen
}

\section{Phasen und Faktoren der Interaktion}

Die Auseinandersetzung mit der Sowjetunion war ein prägender Faktor für die $\mathrm{Ge}$ schichte der deutschen Sozialdemokratie in der Zeit der Weimarer Republik. Über kein anderes Land wurde soviel in der Parteipresse geschrieben, kein anderes Staatswesen war Anlaß für solch intensive Diskussionen, Polemiken und Kämpfe. Die besondere Herausforderung durch die Sowjetunion bestand darin, daß sie das Selbstverständnis der Sozialdemokratie als sozialistischer Bewegung in Frage stellte und zum Leitmodell der um die Vorherrschaft in der Arbeiterbewegung kämpfenden kommunistischen Parteien wurde. Die Konkurrenz der Kommunisten zwang zur Auseinandersetzung mit deren Ideal. Das gilt insbesondere für Länder, in denen die Kommunisten stark waren, vor allem für Deutschland und Frankreich. In diesen Ländern standen die Sozialdemokraten der Sowjetunion denn auch besonders kritisch gegenüber, während etwa die Sozialdemokraten in Österreich und England, wo die Kommunisten politische Randgruppen geblieben waren, das sozialistische Experiment in Rußland wesentlich wohlwollender betrachteten.

Die Phase der intensivsten Interaktion zwischen der deutschen Sozialdemokratie und Sowjetrußland setzte unmittelbar mit der Oktoberrevolution ein und erstreckte sich über sechs Jahre bis zum gescheiterten „Deutschen Oktober" von 1923. Die Machtübernahme der Bolschewiki löste in Deutschland neue Hoffnungen auf einen baldigen Frieden aus. Sie standen zunächst im Vordergrund. Die beginnende Diskussion zwischen Kritikern und Bewunderern der Bolschewiki war eher eine Sache der Theoretiker. Als jedoch nach den Mißerfolgen im Westen und Süden die deutsche Militärmacht und damit das ganze Kaiserreich ins Wanken kam, als um Waffenstillstand gebeten werden und Sozialdemokraten der Zutritt zum Zentrum der Macht gestattet werden mußte, änderte sich das sehr schnell. Nun erwies sich, daß Sowjetrußland gewissermaBen als Antagonist der deutschen Sozialdemokratie geboren worden war. Die Mehrheitssozialdemokraten hatten die Novemberrevolution in Deutschland nicht gewollt, aber sie identifizierten sich voll und ganz mit ihrem Ergebnis: Die SPD wurde die staatstragende Partei der Weimarer Republik, in der sie ihre Vorstellungen von Sozialismus auf parlamentarisch-demokratischem Wege zu realisieren hoffte. Lenin und Trotzki hingegen hatten die Oktoberrevolution ganz auf die Vision von der proletarischen Weltrevolution gegründet. Die Diktatur des Proletariats konnte sich im industriell rückständigen Rußland nach der ursprünglichen Auffassung der Bolschewiki nur halten, wenn sie ihren Siegeszug in die entwickelteren Staaten, allen voran Deutschland, fortsetzte. Es lag auf der Hand, daß Sowjetrußland das seine dazu tun mußte, um diese seine vermeintliche Existenzvoraussetzung in Deutschland zu realisieren. Der ideologische Kampf um Demokratie und Diktatur, der unter dem Eindruck der Oktoberrevolution in der deutschen sozialistischen Arbeiterbewegung entbrannte, wurde so überlagert von den zwischenstaatlichen Konflikten, die die sowjetischen Interventionsversuche in die deutsche Innenpolitik auslösten. Bis zum gescheiterten „Deutschen Oktober" von 1923 und der bald darauf verkündeten These Stalins vom "Sozialismus in einem Land" blieb der Versuch des "Revolutionsexports" ein bestimmender Faktor im deutsch-sowjetischen Verhältnis. 
In derselben Periode fand auch die Umgruppierung der politischen Formationen des deutschen und internationalen Sozialismus statt. Lenins Projekt einer neuen, wirklich revolutionären Internationale und der Streit um das Modell "Sowjetrußland“ und die Weltrevolution gaben dabei die entscheidenden Impulse. In Deutschland bildete das Kampffeld dieses konfliktreichen Prozesses die USPD. Erst deren Spaltung machte die Kommunisten in Deutschland zu einer Massenpartei. Zwei Jahre später vereinigte sich die Rest-USPD wieder mit den Mehrheitssozialdemokraten, ein Vorgang, der in der Bildung der Sozialistischen Arbeiter-Internationale im Mai 1923 ein Pendant auf internationaler Ebene fand. Alle Hoffnungen der „Zentristen“ auf eine gleichberechtigte und offene Kooperation mit den Kommunisten waren zuvor enttäuscht worden.

$\mathrm{Zu}$ den entscheidenden Weichenstellungen, die die Intensität der Interaktion zwischen deutscher Sozialdemokratie und Sowjetunion in der ersten Phase ausmachen, gehören schließlich auch der Bruch und die Wiederherstellung der deutsch-sowjetischen Beziehungen.

Weniger spektakulär, aber für die deutsche Sozialdemokratie ebenfalls von nicht zu unterschätzender Bedeutung war die Ansiedlung der menschewistischen Emigranten in Deutschland. Alexander Stein, der bereits vor dem Ersten Weltkrieg nach Deutschland gekommen und hier geblieben war, hatte schon 1917 als Verbindungsmann zwischen den Menschewiki und dem bolschewismuskritischen Teil der USPD fungiert. Weitere Verbindungen hatten Dittmann und Crispien am Rande des zweiten Kominternkongresses aufgenommen, und spätestens seit Martows Auftritt auf dem Parteitag von Halle nahmen die Menschewiki die Rolle der sozialdemokratischen Kronzeugen gegen das bolschewistische Regime ein.

In der zweiten Phase war die unmittelbare Interaktion zwischen der deutschen Sozialdemokratie und der Sowjetunion deutlich schwächer ausgeprägt. Diese Phase deckt sich weitgehend mit den Jahren der Stabilisierung der Weimarer Republik. Stalins Kurs des „Sozialismus in einem Land“ ließ die weltrevolutionären Aspirationen der Sowjetunion in den Hintergrund rücken. Man widmete sich dort verstärkt inneren Problemen. Dazu gehörte nicht zuletzt der Kampf um Lenins Nachfolge. Die innerparteilichen Streitigkeiten, die auch in der KPD ihren Widerhall fanden, wurden von den deutschen Sozialdemokraten als Schwächezeichen des Kommunismus interpretiert. Obwohl Komintern und KPD nicht ohne Erfolge versuchten, mit dem Programm der „Arbeiterdelegationen“ Ausstrahlung und Attraktion des sowjetischen Modells auf das deutsche Proletariat zu erneuern, lag das Hauptgebiet der Auseinandersetzung zwischen der SPD und der Sowjetunion in dieser Periode auf dem Feld der Außenpolitik. Die ideologische Auseinandersetzung um den sowjetischen Weg zum Sozialismus lief daneben in mittlerweile gewohnter Weise weiter, ohne daß sie durch wesentlich neue Impulse gespeist wurde. Was die internationalen Beziehungen betraf, so war es das primäre Ziel der SPD-Mehrheit, die Verständigungspolitik mit dem Westen nicht durch sowjetische Interventionen stören zu lassen - eine Haltung, die beim linken Flügel der Sozialdemokraten wegen des Verdachts antisowjetischer Tendenzen auf Argwohn stieß, der die Linke aber gleichwohl keine Alternative entgegenzusetzen wußte.

In der kurzen Regierungszeit des Kabinetts Hermann Müller und der langen Phase des Niedergangs der Weimarer Republik war die Interaktion zwischen deutscher Sozialdemokratie und Sowjetunion erneut durch scharfe Konfrontation gekennzeichnet. Insbesondere während der sozialdemokratischen Kanzlerschaft verschränkten sich 
ideologische und außenpolitische Konfrontation erneut, wie dies vor allem bei den Vorgängen um den „Blutmai“ von 1929 deutlich wurde. In der KPD verhalfen diese Ereignisse dem Sozialfaschismus-Kurs zum endgültigen Durchbruch. Die neue Komintern-Politik ist in engem Zusammenhang mit dem Beginn der Zwangskollektivierung der Landwirtschaft und der forcierten Industrialisierung in der Sowjetunion zu sehen ein Umbruch, der in der Sozialdemokratie neue Diskussionen über die Rolle der UdSSR für den internationalen Sozialismus auslöste.

Ihre Einordnung in ein Konzept eines umfassenden, auf verschiedenen Wegen zu verwirklichenden Sozialismus blieb jedoch nur ein Gedankenkonstrukt einiger linkssozialdemokratischer Theoretiker. Das zeigt nicht zuletzt die sowjetische Reaktion auf den Vormarsch des Nationalsozialismus in Deutschland. Friedrich Stampfers Versuch, auf dem Umweg der Verständigung mit der diplomatischen Vertretung der UdSSR in Deutschland zu einem Nichtangriffspakt mit den Kommunisten zu kommen, um die Kräfte auf die Abwehr der Bedrohung durch die Machtübernahme der NSDAP konzentrieren zu können, blieb erfolglos. Die Führung der Sowjetunion setzte keinerlei Hoffnungen auf eine Stärkung der deutschen Sozialdemokratie, und so wurden auch die geringen Hoffnungen einiger deutscher Sozialdemokraten auf sowjetische Hilfe im Kampf gegen den Nationalsozialismus enttäuscht.

\section{Perzeption: Das sozialdemokratische Bild der Sowjetunion und sein politischer Rabmen}

Das Bild, das sich die deutsche Sozialdemokratie von der Sowjetunion machte, war in hohem Maße standortgebunden. Die sozialdemokratische Perzeption war beeinflußt durch ideologische Traditionen, innerdeutsche Verhältnisse und die Notwendigkeit, auf die Herausforderung eines sich als sozialistisch verstehenden Staatswesens zu reagieren. Große Bedeutung kam dem altgewohnten Rußlandbild der SPD zu. Der Zar als oberster Reaktionär Europas war schon seit 1848 der Lieblingsfeind der deutschen Linken gewesen, und das blieb bis 1917 so. Aber bei aller Bewunderung für die russischen Revolutionäre erschien der Sozialdemokratie auch die russische Nation als sozioökonomisch und zivilisatorisch zurückgeblieben. Mehr noch als die Tradition der Rußlandfeindschaft von Marx und Engels - die ja auch das revolutionäre Potential im Zarenreich hoch eingeschätzt hatten - prägte das vom Liberalismus übernommene linear-evolutionäre Fortschrittsdenken den sozialdemokratischen Blick auf Rußland. Der Stand der Industrialisierung und der Arbeiterbewegung, des Rechtsstaates und der Demokratie waren die Indikatoren, die darüber entschieden, welche Sprosse ein Land auf der zum Sozialismus führenden Leiter der historischen Entwicklung erklommen hatte. Rußland kam dabei zweifellos nur ein unterer Platz zu. Ihren klarsten Ausdruck hat diese Einstellung in den Theorien Kautskys gefunden, doch entsprach sie auch den vielen, vielen Erfahrungen des zähen Ringens um kleine Fortschritte, die die deutsche Arbeiterbewegung prägten. Der Versuch jedenfalls, in dem rückständigen Agrarland Rußland den Sozialismus zu verwirklichen, erschien aus dieser Perspektive als unmögliches Unterfangen und trug den Bolschewiki den Vorwurf des Voluntarismus und Blanquismus ein.

Diese Kritik setzte aber nicht unmittelbar nach der Oktoberrevolution ein. Die Bolschewiki erschienen vielmehr als die russische Friedenspartei. Dieser Zug dominierte zunächst in dem Bild, das sich die deutsche Sozialdemokratie von ihnen machte. Zwar 
wurde die Zerschlagung der Konstituante heftig kritisiert, doch übte die SPD vom Frühjahr bis Herbst 1918 wieder große Zurückhaltung, ja sie griff sogar die sozialistischen Opponenten der Bolschewiki an. Der Grund dafür war, daß Lenins Partei als einzige politische Kraft in Rußland den Frieden von Brest-Litowsk trug, dessen Inhalt die SPD zwar ablehnte, den sie aber doch einem Wiederaufleben der Kämpfe vorzog. Mit dem deutschen Waffenstillstandsangebot war diese Konstellation hinfällig. Die SPD orientierte sich nach Westen, unterstützte aus außen- und innenpolitischen Gründen den Abbruch der diplomatischen Beziehungen zu Rußland und konnte nun ihrer Bolschewismuskritik freien Lauf lassen, für die - von tagespolitischen Rücksichten unbeeinflußt - das USPD-Mitglied Karl Kautsky mittlerweile die theoretischen Grundlagen geschaffen hatte. Diese Kritik war dabei seit der Novemberrevolution nicht nur eine Auseinandersetzung mit dem sozialistischen Anspruch, den Sowjetrußland für sich reklamierte, sondern auch mit den deutschen Linksradikalen, für die der Bolschewismus zunehmend, und zwar in dem Maße, in dem sie von der deutschen Entwicklung enttäuscht wurden, Modellcharakter erhielt. Dadurch wurde die Vergleichsperspektive auch in der sozialdemokratischen Perzeption zu einem dominierenden Faktor. Im Zentrum der Wahrnehmung stand daher das, was aus sozialdemokratischer Sicht auch für die deutsche Arbeiterklasse von entscheidender Bedeutung war, vor allem das materielle Wohlergehen der Arbeiterschaft und die Demokratie. Zum Maßstab für letztere wurde vor allem die Aktionsfreiheit von Gewerkschaften und Sozialdemokraten gemacht. Verfolgungen von Kirchen und Intellektuellen, Nationalitätenkonflikte und auch die Lage der Bauern, die immerhin die Bevölkerungsmehrheit ausmachten, interessierten die deutsche Sozialdemokratie hingegen kaum.

Welchen Realitätsgehalt konnte die sozialdemokratische Perzeption unter diesen Voraussetzungen erreichen? Peter Lösche hat darauf eine sehr negative Antwort gegeben: „Das Bolschewismusbild der SPD hat sich in den ersten zwei Jahren nach der Oktoberrevolution geformt und blieb bis in die Jahre der Weltwirtschaftskrise ungebrochen. In der Auseinandersetzung mit den deutschen Linksradikalen erstarrte der Bolschewismus für die Mehrheitspartei zu einem Fetisch, [...] so daß die Wandlung des Bolschewismus vom Leninismus zum Stalinismus in ihrer qualitativen Bedeutung nicht recht reflektiert werden konnte. ${ }^{11}$ Tatsächlich waren die Brandmarkung der bolschewistischen Diktatur - nach der Anfangsphase des Bürgerkriegs, wo weniger von der Diktatur als vom "bolschewistischen Chaos“ die Rede war - und der Hinweis darauf, daß sie den Arbeitenden nicht das gebracht habe, was man sich vom Sozialismus versprach, dauerhafte Konstanten der sozialdemokratischen Bolschewismuskritik. Begriffe wie „Fetisch“ und „Erstarrung“ werden dieser Haltung jedoch nicht gerecht. Vielmehr wurde die einmal gefundene Grundsatzposition der Sozialdemokratie durch die weitere Entwicklung der Sowjetunion bestätigt. Daß sich das politische System von einer Parteidiktatur zu einer persönlichen Diktatur entwickelte, wurde in der deutschen Sozialdemokratie durchaus wahrgenommen. Man sah darin - m. E. mit guten Gründen - aber keine qualitative Veränderung, sondern eher eine folgerichtige und zwangsläufige Entwicklung.

Die sozialdemokratische Wahrnehmung der Sowjetunion war, entgegen der zitierten

\footnotetext{
${ }^{1}$ Lösche, S. 250.
} 
Einschätzung, sogar ziemlich genau und differenziert. Dazu trugen ganz wesentlich die menschewistischen Exilierten bei, die eine rege publizistische Tätigkeit in deutschen Partei- und Gewerkschaftsorganen entfalteten. Abgesehen von außenpolitischen Fragen stammt das meiste, was darin über Rußland erschien, aus menschewistischen Federn. Die emigrierten Menschewiki kannten die politischen Akteure und die sozialen Verhältnisse der Sowjetunion aus eigenem Erleben. Sie sorgten, unter anderem durch ihr Informationsbulletin „RSD“, für einen steten Fluß von Übersetzungen von Nachrichten auch entlegenerer sowjetischer Zeitungen, die die sozialen Verhältnisse kritisch beleuchteten, und erhielten von ihren Vertrauensleuten im Lande ungefilterte Informationen und Stimmungsberichte. Dabei legten die menschewistischen Autoren trotz ihres eindeutigen Standpunktes ein bemerkenswertes Bemühen um Sachlichkeit und Präzision an den Tag. So war ein "Vorwärts"-Leser mit Sicherheit besser über die Sowjetunion informiert als einer der Hugenbergpresse, die unseriöse Sensationsmeldungen über die Sowjetunion verbreitete, aber wohl auch besser als die Leser seriöser bürgerlicher Zeitungen, die, selbst wenn sie Korrespondenten in Moskau stationiert hatten - was der sozialdemokratischen Presse ja von der Sowjetregierung verweigert worden war-, zumeist nur in vergleichsweise geringem Umfang über die Sowjetunion berichteten ${ }^{2}$.

Bei der Kritik an der Sowjetunion standen die zentralen, vom Parteivorstand beeinflußten Organe wie vor allem der „Vorwärts“ oder die "Sozialdemokratische Parteikorrespondenz" in vorderster Linie. Publikationen des linken Flügels hielten sich in dieser Hinsicht stärker zurück. Jedoch hatte die Parteilinke, die sich nach der Wiedervereinigung von SPD und USPD neu formierte, keine grundsätzlich andere Sicht der Sowjetunion als die Mehrheit. Bezeichnend für sie war eher eine Art „Anti-Antibolschewismus“, am prägnantesten veranschaulicht durch Paul Levis Aussage, er wolle kein Urteil über den Terror in der Sowjetunion abgeben, um nicht als Antibolschewist zu gelten ${ }^{3}$. Erst unter dem Eindruck der Weltwirtschaftskrise, des sowjetischen Fünfjahresplans und der daran anknüpfenden Theorien Otto Bauers kam ein Teil der sozialdemokratischen Linken zu der Ansicht, daß sich die Sowjetunion auf ihrem spezifischen Weg zum Sozialismus befinde, wobei es sie zu unterstützen gelte.

Ganz neu war dieser Gedankengang allerdings nicht. Er war nur die Anwendung einer alten Denkfigur auf eine neue Entwicklung, nämlich der Idee, für Rußland könnten andere politische Maßstäbe gelten als für Westeuropa. Interessanterweise hat als einer der ersten Friedrich Stampfer die Ansicht vertreten, in Rußland könnten möglicherweise zuerst grundlegende gesellschaftliche Umwälzungen vorgenommen werden, bevor die Demokratie eingeführt werde 4 . Ähnlich argumentierte die "zentristische" Richtung des internationalen Sozialismus, die sich zeitweilig in der IASP organisierte. Man machte den russischen Kommunisten nur zum Vorwurf, daß sie ihre Erfahrungen anderen sozialistischen Parteien aufdrängen wollten, nahm aber davon Abstand, ihre politische Praxis in der Sowjetunion zu kritisieren. Kritiker dieser Haltung haben stets darauf aufmerksam gemacht, daß damit die Demokratie als Prinzip in Frage gestellt werde. Die Berechtigung ihrer Einwände zeigte sich im Scheitern aller „zentristischen“

\footnotetext{
${ }^{2}$ Müller, Deutsch-sowjetische Beziehungen, S. $13 \mathrm{ff}$.

${ }^{3}$ Siehe oben S. 232.

${ }^{4}$ Siehe oben S. $39 \mathrm{f}$.
} 
Versuche, den prinzipiellen Gegensatz von Demokratie und Diktatur durch Kompromißlösungen zu überbrücken.

\section{"Fernwirkungen"}

Der Einfluß der sowjetischen Entwicklung auf die deutsche Sozialdemokratie blieb nicht auf eine theoretische Auseinandersetzung beschränkt. An einigen Punkten des hier betrachteten Geschichtsabschnitts hatte sie eine bedeutsame Orientierungsfunktion mit wichtigen politischen Folgen.

Von ganz erheblicher Bedeutung für die Politik der Mehrheitssozialdemokratie war das sowjetische Beispiel in der Zeit der deutschen Revolution. Rußland war für die SPD ein warnendes Beispiel für die katastrophalen Folgen einer linksradikalen Politik. Die Errichtung einer Räterepublik müsse auch in Deutschland Bürgerkrieg, wirtschaftlichen Zusammenbruch und allgemeines Chaos zur Folge haben, lautete ihr ceterum censeo in dieser Zeit. Das „Ordnungsbündnis“ Ebert-Groener war eine Folge dieser Furcht. Das hat schon Peter Lösche herausgearbeitet, wenngleich er verkennt, daß "Chaos" und "Bolschewismus" in der Revolutionszeit als Synonyma gebraucht wurden und die Bedeutung von letzterem keineswegs auf eine zielgerichtete Umsturzpolitik beschränkt war'. Der zweite „Basiskompromiß von 1918“ (H. A. Winkler), das Stinnes-Legien-Abkommen zwischen Gewerkschaften und Unternehmern, wäre aufgrund des gewerkschaftlichen Pragmatismus wahrscheinlich auch zustande gekommen, hätte man das russische Beispiel nicht vor Augen gehabt, doch diente auch den Gewerkschaften der Hinweis auf die katastrophale ökonomische Entwicklung in Rußland als Warnung vor einer überstürzten Sozialisierungspolitik und damit zur Abstützung der Zentralarbeitsgemeinschaft.

Auch wenn Karl Liebknecht bei seiner Proklamation der sozialistischen Republik ausdrücklich auf das russische Vorbild Bezug nahm, bestand die vorherrschende Reaktion der Linken, auf die die mehrheitssozialdemokratischen Argumente zielten, zunächst nicht darin, offensiv die bolschewistische Politik zu verteidigen. Zwar stellte man die vorgebrachten Zusammenhänge in Abrede und verwies auf die Erbschaft von Zarismus und Krieg, doch - so argumentierte etwa Ernst Däumig - wollte man durchaus die "Fehler" der Bolschewiki vermeiden. Worin diese Fehler bestanden, wurde allerdings nie explizit ausgesprochen. Selbst Rosa Luxemburg, die sich darüber tatsächlich Gedanken gemacht hatte, ließ sich ja davon abbringen, sie öffentlich auszusprechen. Auch die USPD-Presse brachte ab 1919 nur noch dürre Meldungen über den Verlauf des russischen Bürgerkrieges und offizielle sowjetische Verlautbarungen; Kritisches über die politische Lage blieb ausgespart. So wurde der Boden bereitet für den Ausgang der zweiten wichtigen Entwicklung in der eine sowjetische "Fernwirkung" bedeutsam war: die Spaltung der USPD.

In diesen Prozeß intervenierten sowjetische Politiker auch ganz unmittelbar, doch ausschlaggebend für die Entscheidung der breiten Mitgliedermassen, die in einer Urabstimmung über den Beitritt zur dritten Internationale zu Wort kamen, war weithin die Einschätzung Sowjetrußlands und der weltrevolutionären Perspektiven. Die steigende

${ }^{5}$ Lösche, Bolschewismus, S. 168. 
Sympathie für Sowjetrußland stand,wie Robert F. Wheeler herausgestellt hat, in direktem Zusammenhang mit der Enttäuschung der Linken über den Gang der Dinge in Deutschland. Sie kam daher erst mit einem gewissen zeitlichen Abstand zur Oktoberrevolution zur Wirkung. Ihren Höhepunkt erreichte sie während und unmittelbar nach dem polnisch-sowjetischen Krieg. In weiten Kreisen der Arbeiterschaft herrschte eine eigentümliche Mischung von Gefühlen der Solidarität mit den Verteidigern der russischen Revolution, aber - gerade angesichts eben erlittener eigener Niederlagen, etwa der der Roten Ruhrarmee - auch eine vage Hoffnung auf die zeitweilig vermeintlichen sowjetischen Sieger. Durch die mobilisierende Wirkung der Aktionen gegen Waffentransporte für Polen über deutsches Gebiet wurde dieses Gefühl bestärkt. Weil sie darin eine Entscheidung für Sowjetrußland sah, entschied sich die Mehrzahl der aktiven USPD-Mitglieder für den Beitritt zur Komintern und damit für die Verschmelzung mit der KPD. Daß Wilhelm Dittmann das in der USPD herrschende Schweigen über die tatsächlichen sowjetischen Zustände brach, konnte daran nichts mehr ändern.

Seinen tieferen Grund hatte dies darin, daß hier zwei unterschiedliche Wahrnehmungsweisen vorlagen. Am sinnfälligsten zeigte sich dies an der Begegnung der vier USPD-Delegierten zum zweiten Komintern-Kongreß mit den deutschen Auswanderern in Kolomna. Alle vier hatten die heruntergekommene Fabrik dort besichtigt und die Klagen ihrer Landsleute gehört, und Stoecker und Däumig haben Dittmanns Angaben auch nicht bestritten. Dennoch kamen die Delegierten zu völlig kontroversen Einschätzungen Sowjetrußlands. Aber während die Kritiker auf die triste Gegenwart hinwiesen, hatten die Anhänger des sowjetischen Modells die Zukunftshoffnungen vor Augen, die die russische Revolution in ihnen erweckt und die eigene deutsche Revolution nicht eingelöst hatte. Bezeichnenderweise waren es vor allem die jungen, erst durch die Revolution politisierten USPD-Mitglieder ohne gewerkschaftliche oder parteipolitische Erfahrung, die zur Dritten Internationale tendierten und die revolutionäre Naherwartung ihrer Propagandisten teilten ${ }^{6}$.

Mit der Spaltung der USPD war der Einfluß der sowjetischen Entwicklung auf die organisatorische Umgruppierung des deutschen Sozialismus noch nicht beendet. Im Verein mit anderen linkssozialistischen Parteien versuchte die USPD zunächst, ein linkes Zentrum zu konstituieren, das die schrittweise Zusammenarbeit aller Strömungen des Sozialismus herbeiführen sollte. Dieses Vorhaben erhielt schon während der Gründung der Wiener Union durch die sowjetische Invasion im sozialdemokratisch regierten Georgien einen schweren Stoß und scheiterte nach dem für die $Z$ weite Internationale und die Komintern nur taktisch motivierten Zwischenspiel der Berliner Konferenz der drei Internationalen Exekutivkomitees aufgrund des Prozesses gegen die Sozialrevolutionäre völlig. Die Desillusionierung der Zentristen über die Möglichkeiten einer Zusammenarbeit mit den Kommunisten war ein wesentlicher Faktor für die Wiedervereinigung der sozialdemokratischen Parteien in Deutschland und auf internationaler Ebene.

Daß solche wirksamen Einflüsse wie 1920 nicht auf synthetischem Wege zu erzeugen waren, mußte die KPD erfahren, als sie Mitte der 20 er Jahre versuchte, mittels der "Arbeiterdelegationen" einen KP-freundlichen Flügel in den Gewerkschaften und der

${ }^{6}$ Vgl. dazu Wheeler, USPD und Högl, Gewerkschaften und USPD. 
SPD aufzubauen. Zwar hatten die Arbeiterdelegationen eine große Resonanz, der erhoffte politische Ertrag blieb jedoch aus. Erst als der Kontrast zwischen Weltwirtschaftskrise im Westen und industriellem Aufbau im Zeichen des Fünfjahresplans dem Sowjetmythos neuen Glanz verlieh, wurde die Sowjetunion wieder zu einem Orientierungsthema für innerhalb der Sozialdemokratie. Allerdings spielte bei der Abspaltung der SAP die Rußland-Diskussion bei weitem keine so bedeutende Rolle wie bei der Spaltung der USPD.

\section{Kampfplatz Sowjetunion: Der Streit zwischen SPD und KPD}

Die Existenz einer großen kommunistischen Partei, die sich vollständig mit der Sowjetunion identifizierte, auch von ihr abhängig war und mit der Sozialdemokratie um den Einfluß auf die Arbeiterschaft konkurrierte, war für die letztere ein ständiger Antrieb zur Auseinandersetzung mit der Sowjetunion. Während die Kommunisten auf das leuchtende Vorbild des Arbeiter- und Bauernstaates verwiesen, bemühten sich die Sozialdemokraten, diese Behauptungen zu widerlegen und neigten dazu, sich sowjetische Mißerfolge auf dem eigenen politischen Konto gutzuschreiben. So charakterisierte der „Vorwärts" die Einführung der NEP 1921 als Kapitulation der russischen Kommunisten vor dem Kapitalismus und Bestätigung der reformistischen Politik der SPD.

Zumeist reagierte die Sozialdemokratie allerdings defensiv auf die KPD-Propaganda, wie dies besonders bei den Arbeiterdelegationen und der Fünfjahrplan-Kampagne deutlich wurde. Offensive Kampagnen gegen die KPD mit "sowjetischen Themen" führte die SPD eigentlich nur zweimal: einmal, als sie die eigentlich anderen Motiven entspringenden Enthüllungen der geheimen militärischen Zusammenarbeit zwischen Reichswehr und Roter Armee immer mehr gegen die Kommunisten zuspitzte, und zum zweitenmal, als sie zur Abwehr des kommunistischen Volksbegehrens gegen den Panzerkreuzer A die Kampagne gegen den "Sowjetmilitarismus" entfesselte.

Allerdings stellte die oben angesprochende laufende Berichterstattung der sozialdemokratischen Presse einen ständigen Angriff auf das rosige Bild von der Sowjetunion dar, das die KPD zu zeichnen versuchte.

\section{Die außenpolitische Stellung zur Sowjetunion}

Etwas überspitzt formuliert könnte man sagen: Die erste außenpolitische Entscheidung, die die deutsche Sozialdemokratie entscheidend mit beeinflußt und zu verantworten hatte, war der Abbruch der diplomatischen Beziehungen zu Sowjetrußland im November 1918. Für diese Entscheidung war zum einen die zunehmende revolutionäre Agitation ausschlaggebend, die von der sowjetischen Botschaft ausging, zum anderen aber auch der Versuch, sich das Wohlwollen der Westmächte und damit günstige Friedensbedingungen zu erwerben. Diese doppelte Option gegen die bolschewistische Revolution im Inneren, für den Primat der Westorientierung in der Außenpolitik blieb im Grunde in der gesamten Zeit der Weimarer Republik ausschlaggebend für das außenpolitische Verhältnis der deutschen Sozialdemokratie zur Sowjetunion. Karl Dietrich Erdmanns Aussage, die „innere Staatsräson“ der Weimarer Republik habe auf den liberal-demokratischen Westen verwiesen, aber „die äußere Staatsräson wies in die 
entgegengesetzte Richtung "7, hätte kein zeitgenössischer Sozialdemokrat unterschrieben. In der Zeit der Revolution hätte die östliche Option, wie besonders in dem Fernschreibergespräch Haases mit Čičerin und Radek und in Radeks Rede auf dem Gründungsparteitag der KPD deutlich wurde, den revolutionären Krieg einer deutschsowjetischen Allianz gegen die Entente bedeutet. Die SPD war aber nicht bereit, den Frieden gegen den Krieg und die eben errungene Demokratie gegen die vorgebliche Diktatur des Proletariats einzutauschen, und selbst dem äußersten linken Flügelmann des Rats der Volksbeauftragten, Emil Barth, schien dies kein gangbarer Weg. Bei der USPD-Basis war die Forderung nach Wiederherstellung der Beziehungen zu Sowjetrußland dagegen aus ideologischen Gründen äußerst populär. Die Weigerung der Regierung Bauer im Herbst 1919, sich der alliierten Blockade gegen Rußland anzuschlieBen, machte aber andererseits auch die Grenzen der Bereitschaft der SPD, sich mit den Westmächten zu arrangieren, deutlich. Schon zuvor waren erste vorsichtige Kontakte zu Vertretern der Sowjetregierung aufgenommen worden. Aber erst mit der Konsolidierung der bolschewistischen Herrschaft, dem Ende der alliierten Intervention und dem Eindruck des polnisch-sowjetischen Krieges, dessen Übergreifen auf Deutschland die Mehrheitssozialdemokraten befürchtet hatten, kam es Ende 1920 zu entschiedeneren Initiativen der SPD zur Wiederherstellung normaler Beziehungen zu Rußland. Die Art und Weise, wie dies dann schließlich 1922 am Rande der Konferenz von Genua geschah, mißfiel allerdings der Mehrheit der Sozialdemokraten, weil dies die Westmächte verprellte. Schon hier zeigte sich aber an Stimmen wie der der „Leipziger Volkszeitung", daß die Westorientierung auch nach dem Übergang des linken USPDFlügels ins kommunistische Lager nicht unumstritten war. Die Sozialdemokraten stimmten dem Rapallo-Vertrag zu, waren aber überwiegend der Meinung, nun habe Deutschland seinen Beitrag zu besseren Beziehungen zur Sowjetunion im wesentlichen geleistet. Vorstellungen von "aktiver Außenpolitik“ mit sowjetischem Rückhalt, wie sie in der Ostabteilung des Auswärtigen Amtes ventiliert wurden, waren ihre Sache nicht. Sie strebte im Rahmen einer "demokratischen Außenpolitik" ein umfassendes europäisches Vertragssystem an. Den Rapallo-Vertrag interpretierte sie als ein Element dieses Systems. Die Sowjetunion hingegen sah die Rapallo-Partnerschaft als ein exklusives Verhältnis an und war dementsprechend mißtrauisch gegen die nach der Ruhrbesetzung beginnende deutsche Verständigungspolitik mit dem Westen, die ihr ihren RapalloPartner zu entfremden drohte. Die SPD interpretierte dies als Folge einer imperialistischen Politik in zaristischer Tradition, die darauf aus sei, die europäischen Mächte gegeneinander auszuspielen. Zumal nach der Welle der diplomatischen Anerkennungen des Jahres 1924 schienen ihr bezüglich der Sowjetunion alle außenpolitischen Aufgaben erledigt - bis auf eine, die man nur in Moskau lösen konnte, aber nicht wollte: den sowjetischen Beitritt zum Völkerbund.

Geradezu als Exekutor ihrer ureigenen Westpolitik erschien der SPD Außenminister Stresemann, den sie, obwohl zumeist als Oppositionspartei, bei allen entscheidenden Schritten seiner Verständigungspolitik unterstützte. Zugleich wandte sie sich vehement gegen Moskauer Störversuche und Abstriche bei der Westpolitik aus Rücksicht auf die Sowjetunion. Stresemanns ausbalancierende Vereinbarungen, das Handelsabkommen

\footnotetext{
${ }^{7}$ Erdmann, Ost- und Westorientierung, S. 137.
} 
mit der UdSSR von 1925 und den Berliner Vertrag, nahm sie hin; von ihr selbst wäre die Initiative wohl kaum ausgegangen. Immerhin begrüßte der Teil des linken SPD-Flügels, für den die "Leipziger Volkszeitung" sprach, diese Verträge. Die Linke begleitete die Westpolitik aus Angst vor antisowjetischen Akzenten mit Mißtrauen, hatte aber selbst keine alternative Konzeption vorzuweisen. Das hing nicht zuletzt mit einem gewissen marxistischen Dogmatismus zusammen, wie er in Reinkultur von Paul Levi vertreten wurde. Für ihn war nahezu jeder außenpolitische Vertrag, den die Weimarer Republik abgeschlossen hatte, des Teufels. Unermüdlich betonte er, die einzig richtige sozialistische Außenpolitik bestehe in der Stärkung des Proletariats. Welche Folgen das für das Verhältnis zu anderen Staaten haben sollte, erklärte er allerdings nicht.

$\mathrm{Daß}$ die Sozialdemokraten im Verhältnis zur Sowjetunion als „Falken“ erscheinen, hängt auch mit ihrer besonderen Allergie gegen sowjetische Einmischungsversuche in deutsche Angelegenheiten zusammen, die ja ihrer Konkurrenzpartei KPD zugute kamen und die die von ihnen hauptsächlich getragene Republik unterminierten. Da sie zudem auf die Sowjetunion keinerlei außenpolitische Hoffnungen setzte, galten ihr diplomatische Rücksichten in solchen Fällen nichts, wie etwa die Durchsuchung der sowjetischen Handelsmission 1924 zeigte. Auch bei der innenpolitisch motivierten Enthüllung der militärischen Zusammenarbeit zwischen Reichswehr und Roter Armee bildeten eventuelle Belastungen des deutsch-sowjetischen Verhältnisses kein Hemmnis.

Auch wenn die SPD stets im Einklang mit der SAI jegliche Intervention gegen die Sowjetunion ablehnte und beispielsweise den Abbruch der diplomatischen Beziehungen durch die britische Regierung 1927 kritisierte, war der Mangel an außenpolitischen Berührungspunkten mit der Sowjetunion stets konfliktträchtig. Das zeigte sich, als die SPD 1928 wieder Regierungsmacht übernahm. Obwohl die „Izvestija“ um Verständigung warben und die Sowjetunion, begleitet vom höchsten Lob des „Vorwärts“, erste Schritte zu einer Politik der kollektiven Sicherheit machte, waren die deutsch-sowjetischen Beziehungen zur Zeit der Regierung Müller schweren Belastungen ausgesetzt. Da sich die Regierung ganz auf das Verhältnis zum Westen konzentrierte, trat in den Beziehungen zur Sowjetunion die Abwehr der Unterstützung der KPD stark in den Vordergrund, zumal sich deren Politik im Zuge des „Sozialfaschismus“-Kurses radikalisierte. Der „Blutmai“ von 1929 für den die Hauptverantwortung auf den sozialdemokratischen Polizeipräsidenten von Berlin, Zörgiebel, fiel, tat ein übriges. Schließlich versuchte die SPD, allerdings erfolglos, die deutsch-sowjetischen Beziehungen vom Wohlverhalten der KPD abhängig zu machen.

Die reine ideologische Gegnerschaft zur Sowjetunion war jedoch kein ausschlaggebender Faktor für die Außenpolitik der SPD. Schon Anfang 1920 hatte der "Vorwärts“ erklärt, die deutsche Politik gegenüber Rußland könne nicht davon abhängen, welche Partei dort an der Macht sei. Kautskys 1925 vorgebrachter Vorschlag, die Beziehungen zur Sowjetunion zu nutzen, um dort eine Liberalisierung der politischen Verhältnisse zu erzwingen, verhallte ungehört. Die Außenpolitik der SPD war nicht von ideologischen Sympathien oder Anipathien geprägt, sondern trug durchaus "realpolitische“ Züge. Das außenpolitische Mißverhältnis zur Sowjetunion erwuchs aus deren langjähriger Weigerung, sich in Systeme kollektiver Sicherheit einzuordnen, die der SPD als zuverlässigste Garanten des Friedens erschienen, und der ständigen Mißachtung der inneren Souveränität Deutschlands. Gerade als der erste Grund wegfiel, gewann der zweite um so mehr an Aktualität. 


\section{Sozialdemokratisches Selbstverständnis und Sowjetunion}

Auf die Frage, welche Folgen die permanente Auseinandersetzung mit der Sowjetunion für die deutsche Sozialdemokratie hatte, scheinen die Quellen auf den ersten Blick kaum eine Antwort zu geben. Natürlich markiert die Stellung zur Sowjetunion die Grenze zwischen Sozialdemokraten - oder, wie sie sich, um die Abgrenzung zu betonen, auch nannten, demokratischen Sozialisten - und Kommunisten, was besonders bei der Spaltung der USPD deutlich wurde. Danach spielt die Sowjetunion in der programmatischen Diskussion der Sozialdemokratie jedoch keine Rolle mehr. Bezeichnenderweise war die Sowjetunion auch weniger ein Thema sozialdemokratischer Parteitage (die USPD immer ausgenommen) als der sozialdemokratischen Publizistik, die es um so intensiver bearbeitete. $\mathrm{Da} ß$ von der Sowjetunion keine positiven programmatischen Impulse auf die deutsche Sozialdemokratie ausgingen, ist jedoch insofern verständlich, als dieser der Bolschewismus als ein Produkt historisch rückständiger Verhältnisse erschien. So fielen die Antworten auf die Frage, was die Sozialdemokratie von der Sowjetunion lernen könne, negativ aus. Die tiefste Lehre aus den russischen Verhältnissen sei, so erklärte etwa Otto Wels auf dem Leipziger Parteitag von 1931: „Ohne Demokratie ist der Sozialismus unmöglich". Und der Vorsitzende des Deutschen Holzarbeiterverbandes, Fritz Tarnow, sagte ebenda, das russische Beispiel gebe auf die Frage, wie man eine Industriewirtschaft mit einem Ruck vom kapitalistischen auf das sozialistische System umstellen könne, keine andere Antwort, als daß man es so unmöglich machen könne.

Obwohl sie wenig Greifbares zu enthalten scheinen, geben Wels' und Leiparts Aussagen doch einen wichtigen Hinweis darauf, was die deutsche Sozialdemokratie von der Sowjetunion lernte. In der Weimarer Republik, wo sie erstmals die Chance zu effektiver politischer Mitgestaltung hatte, begann ihr Wandel von einer Partei des „revolutionären Attentismus“, die sich letzten Endes durch die Erwartung des in unbestimmter Zukunft eintretenden, alles verändernden "großen Kladderadatsch“ legitimierte, zu einer sozialen Reformpartei, die versuchte, Theorie und Praxis in Einklang zu bringen. Davon zeugt vor allem die Diskussion über Wirtschaftsdemokratie Mitte der zwanziger Jahre. Die Sowjetunion war in diesem Zusammenhang eine stete Warnung vor dem Rückfall in utopische Vorstellungen, deren Folgen sie in ihrer nackten, entzauberten Gestalt vorführte. Was man von ihr lernen konnte, war, "wie man den Sozialismus nicht machen darf". 